University of Nebraska - Lincoln

DigitalCommons@University of Nebraska - Lincoln

USDA National Wildlife Research Center - Staff

Publications

U.S. Department of Agriculture: Animal and Plant Health Inspection Service

2013

\title{
An analytical framework for quantifying and testing patterns of temporal dynamics in social networks
}

\author{
Elizabeth A. Hobson \\ New Mexico State University, ehobson@santafe.edu \\ Michael L. Avery \\ U.S. Department of Agriculture, michael.I.avery@aphis.usda.gov \\ Timothy F. Wright \\ New Mexico State University, wright@nmsu.edu
}

Follow this and additional works at: https://digitalcommons.unl.edu/icwdm_usdanwrc

Part of the Life Sciences Commons

Hobson, Elizabeth A.; Avery, Michael L.; and Wright, Timothy F., "An analytical framework for quantifying and testing patterns of temporal dynamics in social networks" (2013). USDA National Wildlife Research Center - Staff Publications. 1147.

https://digitalcommons.unl.edu/icwdm_usdanwrc/1147

This Article is brought to you for free and open access by the U.S. Department of Agriculture: Animal and Plant Health Inspection Service at DigitalCommons@University of Nebraska - Lincoln. It has been accepted for inclusion in USDA National Wildlife Research Center - Staff Publications by an authorized administrator of DigitalCommons@University of Nebraska - Lincoln. 


\title{
An analytical framework for quantifying and testing patterns of temporal dynamics in social networks
}

\author{
Elizabeth A. Hobson ${ }^{\mathrm{a}, *}$, Michael L. Avery ${ }^{\mathrm{b}}$, Timothy F. Wright ${ }^{\mathrm{a}}$ \\ a Biology Department, New Mexico State University, Las Cruces, NM, U.S.A. \\ ${ }^{\mathrm{b}}$ U.S. Department of Agriculture, Florida Field Station, Gainesville, FL, U.S.A.
}

\section{A R T I C L E I N F O}

Article history:

Received 24 May 2012

Initial acceptance 20 June 2012

Final acceptance 19 September 2012

Available online 15 November 2012

MS. number: A12-00395R

\section{Keywords:}

association strength

monk parakeet

multiscale approach

Myiopsitta monachus

network formation

social structure

temporal analysis

\begin{abstract}
Change is fundamental to all social systems. Temporal dynamics are critical in understanding how relationships form and change over time but rarely are studied explicitly in animal groups. Social network approaches are useful in describing association patterns and provide promising tools for investigating the dynamics of change in social structure but have rarely been used to quantify how animal associations change over time. In this study, we describe and test a framework for temporal analysis of social structure. We propose an analytical framework of methods that integrates across social scales and comparatively analyses change in social structure across multiple types of social association. These methods enable comparisons in groups that differ in size and are flexible to allow application to weighted and unweighted networks, where ties can be directed or undirected, and relationships can be symmetric or asymmetric. We apply this analytical framework to temporal social network data from experimentally formed captive groups of monk parakeets, Myiopsitta monachus, to both evaluate our analysis methods and characterize the social structure of this species. We compared dynamics of dyadic network formation, ego network formation and global network stabilization patterns across neutral, affiliative and agonistic associations. We found that social structure of captive monk parakeets formed and stabilized over a short period, but patterns differed by social association type. We also found evidence for consistency in the temporal dynamics of formation and stabilization of social structure between replicate social groups. Our analysis methods successfully identified change in social structure that corresponded well with qualitative observations. This framework is likely to be useful in characterizing patterns of temporal dynamics in social structure in longitudinal data in wide variety of social systems and species.

(C) 2012 The Association for the Study of Animal Behaviour. Published by Elsevier Ltd. All rights reserved.
\end{abstract}

Change is fundamental to all social systems. Social associations vary over time as individuals are incorporated into social groups through birth and immigration and leave through emigration and death. This process can be somewhat predictable in cases of longlived species with stable social groups. In contrast, this process is less predictable when mortality rates are higher or when individuals alter their group membership more frequently, as in the case of species with high fission-fusion dynamics. The importance of an individual's social associates can also be context dependent and can shift with changes in developmental, environmental or social conditions (Hinde 1976a; Sapolsky 2005). Once formed, relationships among individuals are unlikely to remain static, and the

\footnotetext{
* Correspondence: E. A. Hobson, Biology Department MSC 3AF, New Mexico State University, Las Cruces, NM 88003, U.S.A.

E-mail address: emoseman@nmsu.edu (E. A. Hobson).
}

consistency or stability of relationships is important to consider when characterizing social patterns (Hinde 1976a).

\section{Temporal Dynamics of Social Networks}

Temporal dynamics are key to understanding how relationships form and change over time but are rarely studied explicitly in animal groups (Whitehead 2008; Krause \& Ruxton 2010). One method for analysing social relationships is social network analysis (Wasserman \& Faust 1994; Wey et al. 2007; Croft et al. 2008). Social network analysis is a flexible, model-free technique applicable to a wide range of social situations. It provides methods for quantifying relationships and how individuals fit into the social landscape on a global scale. Although social network analysis provides promising tools for investigating the dynamics of change in social structure, it has rarely been used to quantify how animal associations change over time. Where temporal dynamics of social 
structure have been studied, the focus has generally been on gross structural patterns obtained when data are aggregated over long timescales. For example, social network structure in some groups changes with season (Hamede et al. 2009; de Silva et al. 2011), reproductive state (Fischhoff et al. 2007; Sundaresan et al. 2007; Patriquin et al. 2010), individual attractiveness (Oh \& Badyaev 2010) and resource availability (Henzi et al. 2009; Foster et al. 2012). Other studies have found evidence for stable social associations across years (e.g. Silk et al. 2006a; Mitani 2009) and correlations with individual fitness across years (McDonald 2007; Ryder et al. 2008). Data in these analyses were generally aggregated over relatively long periods such as months or years. This type of data aggregation is a snapshot method of comparing static networks to one another and does not allow for detailed exploration of dynamic change. Studies of the fine details of network change are much less common (but see Blonder \& Dornhaus 2011).

Methods have been developed to study temporal dynamics in human social networks to predict patterns of formation of social ties. Exponential random graph modelling or $p^{*}$ models (reviewed in: Wasserman \& Pattison 1996; Anderson et al. 1999; Robins et al. 2007) can be used to determine the probability of the occurrence of network ties based on predictor values (Robins et al. 2007). However, parameters for these methods are complex, results can be difficult to interpret and the models are best used to differentiate between tie occurrence based on peer influence or self-driven selection (Borgatti 2010). To quantify and test how patterns of social associations and structure change over time, methods that are more easily interpreted and that can be generalized to a broader range of study questions are needed (Borgatti 2010). In addition, most of the development of tools in the social sciences has focused on dichotomous ties between individuals, where a tie is either present or absent; much less work has focused on development of statistical analysis of continuous weighted tie strengths or valued relations (Robins et al. 1999). There has been a recent push to move towards use of continuous measures of dyadic association strength in network studies, especially in nonhuman animals (see Lusseau et al. 2008; Croft et al. 2011).

Two major aspects of the dynamics of social networks that have received little attention are (1) analyses of temporal dynamics across different social scales and (2) comparative analyses of dynamics across different association types. First, social structure has generally been examined at a single scale. Representations of social structure are usually constructed based on characteristics of dyadic relationships (Wasserman \& Faust 1994; Whitehead 2008), and then analysis focuses on dyadic relationships (Silk et al. 2006a, b, 2009), individual connections within the network structure (Stanton et al. 2011), or the group's global network structure (Lusseau et al. 2006; Henzi et al. 2009). However, changes in social structure may be better understood by using a scaled approach that considers aspects of temporal dynamics across different social levels. Social networks can be examined at multiple levels to determine how change occurs over time in dyadic relationships, how individuals interact within local social structure, and characteristics of the entire network on a global scale. A multiscale approach could thus provide a more comprehensive perspective on the drivers and characteristics of temporal dynamics in networks (Mucha et al. 2010; de Silva et al. 2011).

Second, analyses of temporal dynamics generally focus on a single type of social association. Associations among individuals may be affiliative, agonistic or behaviourally neutral. There are few comparative studies of social structure dynamics across multiple association types to examine differences in the speed of formation of different types of social structure. For example, previous studies on the temporal dynamics of dominance hierarchy formation solely incorporate information on agonistic associations (Chase 1980;
Chase et al. 2002). Comparisons of temporal dynamics across multiple association types, or a 'multiplex' approach to social structure analysis (Hinde 1976b; Wasserman \& Faust 1994; Hamill 2006; Croft et al. 2008; Mucha et al. 2010), could provide a more comprehensive and comparative perspective on social change. Even in groups structured by aggression, mutual dependencies exist between group members (de Waal 1986). Comparisons across multiple association types are especially important because an individual's connections within a group have been shown to have important fitness consequences. For example, neutral associations, through shared group membership, can increase foraging efficiency in vultures (Coragyps atratus and Cathartes aura; Buckley 1996; Rabenold 1987) and access to other essential resources in Grevy's zebra, Equus grevyi (Sundaresan et al. 2007). Affiliative associations increase reproductive success in wire-tailed manakins, Pipra filicauda (Ryder et al. 2008, 2009), and a mother's affiliative grooming network is positively correlated with infant survival in baboons (Papio cynocephalus; Silk et al. 2009). Agonistic associations, especially in species with linear dominance hierarchies, are often correlated with increased access to reproductive opportunities and the potential to pass rank on to offspring in spotted hyaenas, Crocuta crocuta (Holekamp \& Smale 1991; East et al. 2009). In yellowbellied marmots, Marmota flaviventris, aggressiveness or bullying is positively associated with male reproductive success (Wey \& Blumstein 2012). Studies have also documented that changes in one association type can affect associations in another context. For example, patterns of social associations at one life stage can affect future associations, status and reproductive success in male manakins (Chiroxiphia linearis: McDonald 2007; Pipra filicauda: Ryder et al. 2008, 2009).

Despite previous research, large gaps remain in our understanding of complex sociality in many taxa (Silk 2007), which hampers efforts to understand broader patterns in the evolution of social structure. More detailed understanding of the temporal dynamics of social structure within and across association types could help provide insight into the processes driving complex sociality (Krebs \& Davies 1996; Croft et al. 2008).

\section{Analytical Framework}

In this study, we describe and evaluate a framework to address questions about the temporal dynamics of social structure. We provide methods for quantifying and testing temporal dynamics that integrate across social scales and compare change in social structure across multiple association types for a more holistic view of formation and stabilization patterns over time. This framework is particularly applicable to studies with short-term temporal data sets that are difficult to analyse with more traditional time series analysis methods. We outline analyses in increasing order of social scale, from the micro social scale of dyadic relationships through the macro social scale of global network structure. This framework is built in part on a conceptual model developed by Hinde (1976a) for studying primate social structure using concepts derived from human social sciences. Importantly, this model did not specify analyses that could be used, nor did it explicitly include temporal dynamics, although Hinde stressed that patterns and stability of relationships are important characteristics to include (Hinde 1976a, b). A similar conceptual framework was more recently used to quantify dynamics of elephant social structure through time at three social scales (de Silva et al. 2011), but the study focused on a single association type within a single population and thus lacks the multiplex approach described here.

We build on previous work to propose a framework that incorporates analyses that are broadly applicable to comparisons across multiple association types in a wide range of social systems. 
We preserve Hinde's (1976a) original goal in providing a flexible framework that can be used to accommodate studies of diverse nonhuman species. We expand upon the analyses provided by de Silva et al. (2011) to provide additional methods to compare formation and stabilization patterns from a multiplex perspective that allows for examination of similarities and differences among the temporal dynamics of different social association types. We further increase the flexibility of the framework by incorporating analyses that enable comparisons in groups that differ in size. Our framework is also flexible in that the analyses can be applied to both weighted and unweighted networks, where ties can be directed or undirected, and relationships can be symmetric or asymmetric. These aspects allow for broad application of the framework to species with even highly connected dense networks that are difficult to interpret with binary data, where weighted measures of tie strength are likely to be more informative of dyadic relations. This flexibility also allows for the use of directional ties and asymmetric relations, which are both important in considering affiliative and agonistic associations. Our aim is to bring this analytical framework to broader attention and to expand on analyses that can be used to quantify and test patterns of social structure formation and stabilization across time periods and in different social associations.

The first step in the analytical framework is to quantify dyadic association strengths among all individuals at each time period (Fig. 1a). This step of analysis focuses on dyadic relationships as the basis of social structure because social structure is generally constructed based on pairwise relationships between individuals (Hinde 1976a; Wasserman \& Faust 1994; Whitehead 2008). Changes in continuous measures of each dyadic association are characterized based on the extent with which they fit a linear or other type of relationship (Fig. 1b; for fitting nonlinear relationships, see de Silva et al. 2011). Departures from the general relationship trajectory can be used to determine the extent, timing and magnitude of changes in social associations.

Next, individual or ego network structure is characterized through quantifying individually based network metrics. Individuals interact within the social structure both locally, through direct ties with others, and more globally, through indirect ties with others. Each individual serves as a focal node (ego) and network metrics are quantified for each individual based on how it associates with others (Fig. 1c). Ego network measures, such as degree (the number of social associates) or strength (sum of tie strengths), allow each individual's direct ties to be quantified (Wasserman \& Faust 1994; Barthélemy et al. 2005; Whitehead 2008). Summary network statistics of mean individual ego network measures can be used to determine group-level patterns and to test for differences in social structure formation among groups (Fig. 1d).

The final stage in our analysis of temporal dynamics integrates continuous measures of dyadic association strengths with aspects of local and global social structure to examine network stabilization patterns through time (Fig. 1e). We use matrix permutation (a)

Dyadic associations

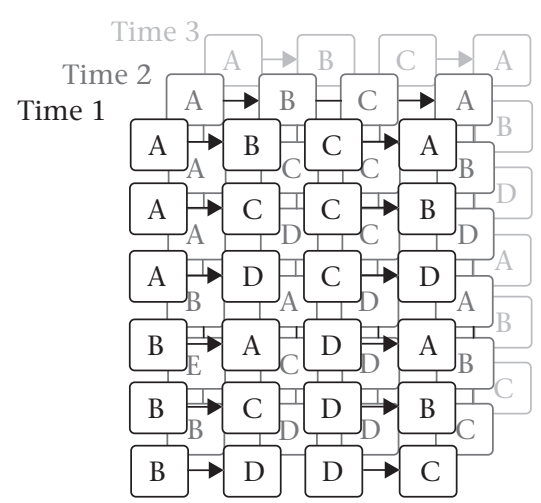

(b)

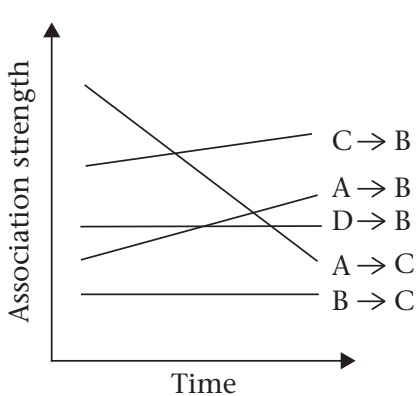

(c)

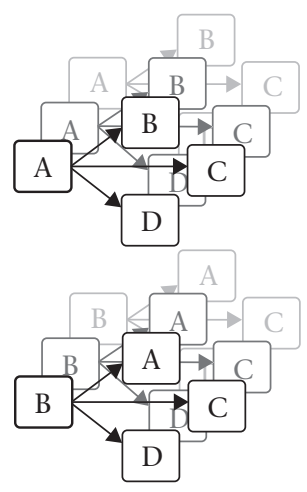

(d)

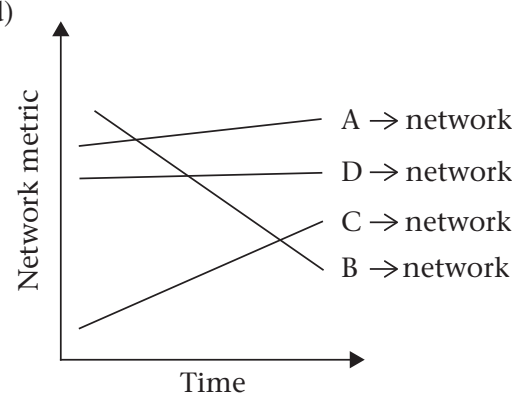

(e)

Global structure
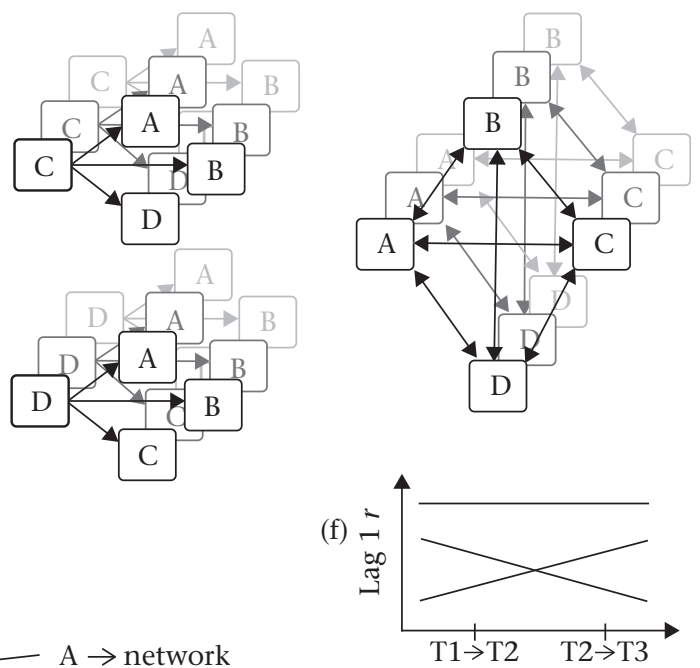

Matrix comparison

(g)

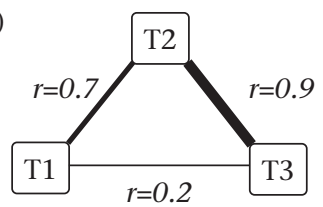

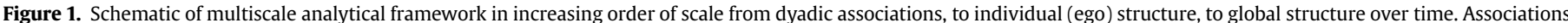

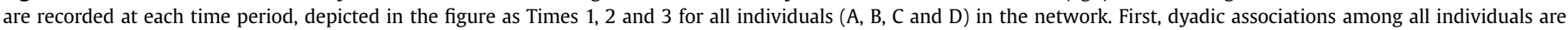

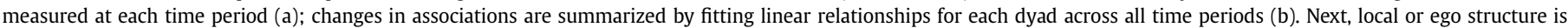

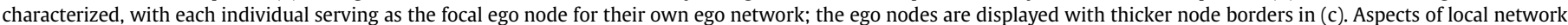

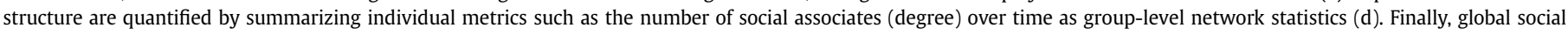

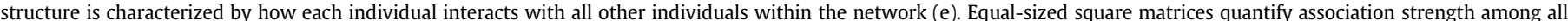

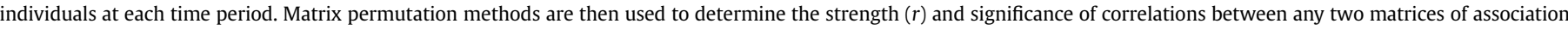

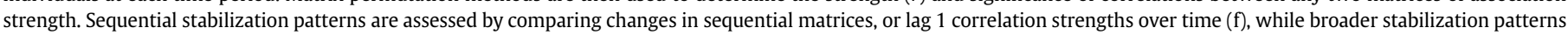

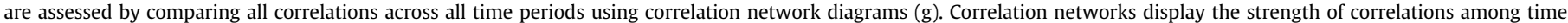

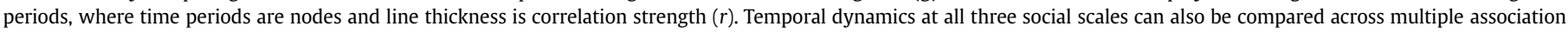
types (not pictured). 
methods to test for the significance and strength of correlations among time periods (Croft et al. 2008). Matrix permutation methods are suitable for nonindependent social structure data (Krackhardt 1988; Croft et al. 2008, 2011) and provide a method for considering both the strength of association between dyads with aspects of more global social structure to serve as the basis for analysis of social stabilization patterns. Matrix methods also allow for comparison among association types, particularly as they preserve the dyadic nature of agonistic associations, thereby allowing comparison with types of dyadic relationships that are not amenable to group-wide hierarchical systems. Matrix methods can accommodate groups with nonlinear dominance hierarchies, or those with undefined, missing or intransitive patterns of aggression, as well as groups with linear hierarchies. In addition, matrix methods can be applied even in cases where individuals do not interact with all other individuals and network connectivity is low because noninteracting dyads receive an association strength of 0 , which would be incorporated into matrix tests. While these matrix methods can be used to compare groups with the same number of individuals, groups that differ in size cannot be compared directly (Croft et al. 2008). Here, we focus on patterns of correlation strength within groups across time to compare temporal dynamics between groups directly. We propose methods to analyse both sequential (correlation between lag 1 sequential time periods; Fig. 1f) and broader (correlation among all time periods) stabilization patterns (Fig. 1g). Sequential stabilization measures can inform about day-to-day changes in social structure, while broader stabilization measures provide perspective on the amount of total change that has occurred over entire study periods, and is most likely to be useful for examining the occurrence of seasonal fluctuations or cycles in social structure stability and the scale and timing of network dynamics (Hinde 1976a).

\section{Study Objectives}

In this study, we have two main goals. First, we describe a framework for analysis of the temporal dynamics of social structure across social scales and association types. We use multiscale analyses of social associations to examine change across social scales, from dyadic associations to global network characteristics. We use a multiplex perspective to compare formation and stabilization patterns across multiple association types to illustrate the applicability of our analytical methodology and to examine differences in patterns among association types.

Second, we use this analytical framework to analyse temporal social network data from the monk parakeet, Myiopsitta monachus, to evaluate our analysis methods and to increase our understanding of the social structure in this species. The monk parakeet is a small parrot native to temperate South America that shows complex social interactions. Individuals nest colonially in communal nest structures, display a high degree of dynamic fission-fusion flocking behaviour, interact with a large diversity of social associates, and engage in affiliative, agonistic and neutral associations (Martín \& Bucher 1993; Eberhard 1998; E. Hobson, unpublished data). We expected that patterns of formation and stabilization of social structure would differ by association type. Based on previous studies representing such diverse taxa as chickens (Gallus domesticus; Chase 1982) and crayfish (Procambarus clarkii; Herberholz et al. 2003), we predicted that agonistic associations, or dominance interactions, would form and stabilize more quickly than would affiliative social structure.

We conduct analyses in increasing order of scale across neutral, affiliative and agonistic social associations. We begin with analyses focusing on the formation of dyadic relationships. We then quantify the formation of local and global network structure from the perspective of each individual. We combine both dyadic relationship strength and network structure to evaluate global patterns of network stabilization. We use our results to gain insight into monk parakeet social structure and to evaluate the utility and generalizability of the analytical framework.

\section{METHODS}

\section{Study Site and Population}

This study was conducted with a population of captive monk parakeets housed at the Florida Field Station of the U.S. Department of Agriculture, National Wildlife Research Center, Gainesville, FL, U.S.A., during June-August 2008. Prior to our study, parakeets were housed in groups of one to six individuals per cage and were able to vocally interact with all other individuals; while some were in visual contact, direct physical contact between individuals in different cages was not possible. We randomly selected individuals without replacement to create two replicate social group treatments: group $1(N=21)$ and group $2(N=19)$. While the replicate experimentally formed groups represent a novel social situation because the individuals had never before interacted with each other as a group, not all dyadic associations among individuals were novel due to prior housing conditions. Random group assignment resulted in the formation of mostly novel dyadic associations: only 3\% (group 1) and $6 \%$ (group 2) of dyads were composed of birds housed together during the 8 months preceding the study.

We marked each parakeet with a unique colour combination applied with nontoxic marker (Sharpie ${ }^{\circledR}$, Sanford, Newell Rubbermaid Co., Oak Brook, IL, U.S.A.) to enable identification of individuals at more than a $270^{\circ}$ radius. Marks did not appear to affect social interactions or individual recognition; pairs allopreened each other within $5 \mathrm{~min}$ of being marked (E. Hobson, personal observation).

We introduced each social group independently into a large $2025 \mathrm{~m}^{2}$ outdoor seminatural flight pen to conduct social experiments. The group not under study remained in their maintenance cages with visual and acoustic separation from the group in the flight pen. The flight pen was a fully contained wire-mesh aviary exposed to natural weather patterns that contained numerous trees and bushes and three food and water stations. Test groups were also exposed to the perception of predation pressure. Because of the large size and complex habitat of the flight pen, individuals could actively avoid each other if desired (E. Hobson, personal observation). Each replicate social group occupied the flight pen for 24 days. Because some of our analyses required the same number of time periods to compare between replicate social groups, and because sampling effort varied slightly between groups due to scheduling and weather, we pooled observations into eight 3-day time periods to enable comparison between groups 1 and 2 . Pooling data on a 3-day scale allowed us to balance our desire to sample detailed social structure with the need for enough observations to represent social structure accurately. Observations of social behaviour were collected by one to four observers in blinds between 0700 and 1900 hours. Data were entered directly into Palm devices or narrated onto an audio recorder and then processed and stored in a relational database (Access 2003, Microsoft, Inc.). All activities conducted during this study were approved by the New Mexico State University Animal Care and Use Committee (protocol number 2006-027).

\section{Quantification of Association Strength}

Observations of three types of associations were collected to generate social networks for three association types: affiliative, 
agonistic and neutral. Because of our captive situation, we used a hybrid observation sampling protocol that combined scan sampling of associations and all-event sampling of interactions (Whitehead 2008). We used scan survey samples to collect information on the location of each individual within the flight pen (Whitehead 2008) to assign each individual to a flock. We also recorded data on the identity of each individual's nearest neighbour and collected directed aggressive interaction data using allevent sampling procedures during our scan samples. We quantified neutral, affiliative and agonistic dyadic association strengths for each of the eight time periods and for both groups to examine temporal dynamics.

Neutral associations were quantified using the 'gambit of the group' to define associations by group membership (Whitehead 2008), here using data on shared flock membership. This measure was the broadest definition of association used in the study and included the most data on the largest variety of social associations and interactions. We used both spatial and temporal proximity to determine shared flock membership, where a flock was defined as all birds found within the same $10 \times 10 \mathrm{~m}$ quadrat or perching location during the same scan sample. When cohesive flocks of individuals spanned multiple quadrats, we assigned location to the quadrat occupied by the majority of the flock. Data on flock membership was pooled into 10 min sampling periods, and any individuals observed in the same flock at any point during a sampling period were considered associated. We avoided nonindependence issues (Sundaresan et al. 2009) even with short sampling periods because individuals were able to change flock membership quickly; an individual could traverse the length of the flight pen in less than $10 \mathrm{~s}$ (E. Hobson, personal observation). Neutral association strength was calculated using the simple ratio index (SRI) in SOCPROG v.2.4 (Whitehead 2009a, b), which is a symmetric measure of dyadic association strength. This measure is often used in captive studies such as ours when individual detection probabilities are not affected by whether individuals are grouped with others during sampling (Cairns \& Schwager 1987; Ginsberg \& Young 1992; Whitehead 2008). To determine whether neutral associations exhibited nonrandom patterns, we permutated observed association matrices following Manly (1995) and Bejder et al. (1998) with modifications introduced in Whitehead (1999) and Whitehead et al. (2005). For this test, $P$ indicates the proportion of randomly generated associations that were smaller than the observed associations, with a large $P(>0.95)$ providing evidence of nonrandom associations (Bejder et al. 1998; Whitehead 2009a). We used SOCPROG to permute associations within sampling periods with 10000 permutations and 1000 flips per permutation. We chose 10000 permutations because values of $P$ stabilized with this number of permutations (E. Hobson, unpublished data, following recommendations from Bejder et al. 1998).

Affiliative association strength was quantified using nearestneighbour identity, defined by proximity within a flock. Unlike other species such as primates, monk parakeets generally only display directed affiliative behaviours, such as allopreening, towards one primary associate, resulting in a highly disconnected network (E. Hobson, unpublished data). However, individuals may have important relationships without physically interacting, and relationships among individuals that rarely interact may be more appropriately characterized by defining associations rather than through directed behavioural interactions (Whitehead 2008). Consequently, we used nearest-neighbour association data to quantify affiliative association strengths. Nearest-neighbour identity is an asymmetric measure of dyadic association strength. Because the amount of nearest-neighbour data collected varied between time periods due to weather and differences in observation effort, we standardized nearest-neighbour count data by the total number of nearest-neighbour observations in each time period to return the percentage of total association observations attributed to each dyad. This method is valid in our circumstances because we had approximately the same probability of observing all individuals during all sampling periods due to the captive conditions.

Agonistic associations were quantified using directed aggressive interactions between individuals. We recorded aggressive behaviours (displacements, threats, bites and chases) using all-event sampling (Whitehead 2008) and identified the aggressor and recipient of aggression for each interaction. We used SOCPROG to quantify dyadic dominance scores for each pair of birds, which measures the observed proportion of wins corrected for the chance occurrence of the outcome (de Vries et al. 2006). Dyadic dominance is an asymmetric measure of dyadic association strength. We used a measure of dyadic dominance status (Drews 1993) rather than ranking individuals in a dominance hierarchy. Dominance rank of individuals in the group could not be directly compared to changes in neutral and affiliative dyadic relationships because individual relationships in these two contexts cannot be organized in a similar ranked manner within the group. We specifically chose to use dyadic dominance because it quantifies aggressive interactions on a dyadic scale, which allows direct comparison to patterns of dyadic relationship strength dynamics in neutral and affiliative contexts.

For each association type, observations were pooled so that dyadic association strength was quantified for each of the eight time periods. Patterns of formation and stabilization of association types were tested between replicate groups 1 and 2 to determine consistency in the temporal dynamics of social patterns. However, the data collected for different association types cannot be considered independent within groups. For example, all affiliatively and agonistically interacting individuals required close proximity; individuals had to be categorized as within the same flock to have the possibility of associating affiliatively or agonistically; thus, all affiliative and agonistic associations were nested within neutral association data. Similar nonindependence issues are likely to occur in comparisons of multiplex associations in other studies, suggesting that care should be taken before statistically comparing across association types. In our case, the nonindependence among within-group data sets precluded statistical comparisons within groups across different association types. Thus, we focus statistical tests on comparisons between replicate social groups.

\section{Formation of Dyadic Associations}

We used the three continuous measures of neutral, affiliative and agonistic association strength detailed above to quantify how dyads associated with one another at each time period (Fig. 1a). We then determined the extent to which change in the dyadic association over time could be described linearly by fitting a regression of time against association strength for each dyad (Fig. 1b). We evaluated each relationship to determine statistical significance, slope, mean square error (MSE) and residuals. The slope of the line indicates the trajectory of each association over time. For neutral and affiliative associations, a positive slope indicates a strengthening association and a negative slope indicates a weakening association. For agonistic associations, a nonzero slope indicates a relationship that is becoming more asymmetric over time; positive slopes indicate that one individual is becoming more dominant over another and negative slopes indicate situations where one individual is becoming more subordinate to another. Dyads with agonistic associations that have nonzero slopes are expected to diverge: as A becomes more dominant (positive slope), B should become more subordinate (negative slope). We quantified the percentage of dyads with statistically significant association 
trajectory regressions and then calculated the percentage of significant trajectories that had positive and negative slopes. A slope near 0 may indicate either a dyadic association that is relatively stable through time, or one that is poorly described with a linear function. To differentiate between these alternatives, we examined the mean square error of the regression lines to determine goodness of fit regardless of the slope of the line. To determine the time periods when associations differed the most from the linear relationship, we quantified the average size of residuals for all dyads at each time period for each association type and replicate group.

\section{Formation of Ego Network Structure}

For analysis of how individuals interact within their local social structure, we quantified aspects of ego network structure to compare patterns of formation across affiliative, agonistic and neutral association types (Fig. 1c). While the dynamics of weighted measures can be used to examine the formation of ego network structure, direct comparisons of weighted measures across multiple association types and groups that differ in size can be difficult to interpret. To facilitate comparison among association types and between groups, we simplified our weighted measures of dyadic association strength to binary tie scores representing the presence or absence of a tie between individuals. Although recent work has advocated for the use of weighted ties over binary ties (Lusseau et al. 2008; Croft et al. 2011), there are some cases where binary data can provide useful information about basic social structure (Croft et al. 2011). In particular, weighted tie strengths are likely to be particularly problematic if the goal is direct comparison of social structure across multiple association types such as comparisons of affiliative and agonistic associations. For example, a high score for an affiliative association indicates a strong preferential association between the two individuals, but a high score for an agonistic association indicates that one individual is highly aggressive towards another. Unless the research question focuses solely on the frequency of social contact (such as in disease transmission studies; e.g. Hamede et al. 2009), directly comparing these different association strengths can result in an illogical comparison that is not biologically meaningful. Simplifying association strength to binary data allows comparison of more basic social structures (such as the number of each individual's associates) across multiple association types and facilitates standardizing network measures across groups of different sizes (T. Opsahl, personal communication).

Because our captive study system promoted high rates of association and interaction and resulted in relatively dense social networks, we focused on degree as our measure of network centrality. Degree in its most general sense is simply a count of the total number of social associates for each individual and is a measure of the extent of an individual's ego network (Wasserman \& Faust 1994). Binary degree allowed for straightforward comparison of how the number of social associates changed over time. Other measures of network centrality (e.g. betweenness, closeness) and network structure (e.g. path length, clustering coefficient, modularity) could also be quantified and may be useful in other situations (Wasserman \& Faust 1994; Wey et al. 2007; Croft et al. 2008; Whitehead 2008). Here, we focused on degree centrality as a network metric because it was likely to be biologically meaningful to the individuals and because it facilitated comparison of basic network features between groups and across association types. We used the $\mathrm{R}$ package thet v.3.0.5 for all social network metrics (Opsahl 2009, 2011).

We used binary data from shared flock membership (SRI), nearest-neighbour identities and aggressive behavioural interactions to determine whether each individual interacted with other individuals during each of the eight time periods. For each association type, individuals received a tie score of ' 1 ' (tie present) if they were observed associating at least once during a time period, whereas individuals that never associated during that time period received a tie score of ' 0 ' (tie absent). Because observations of affiliative and agonistic associations and interactions were made within the same groups and over the same time spans, we are convinced that the absence of ties is biologically meaningful (Croft et al. 2011). We analysed unfiltered neutral networks (no ties excluded) because we were interested in comparing basic structures across association types. We also filtered neutral networks so that individuals received a ' 1 ' (tie present) if they were observed in the same flock during more than $5 \%$ of the sampling periods in each time period. These filtered neutral networks allowed us to exclude observations that may reflect misidentifications or very rare associations that may not be biologically meaningful. Application of these criteria also allowed us to compare filtered and unfiltered networks to examine the effect of data exclusion and evaluate whether neutral networks were biologically meaningful.

To determine local network formation patterns, we quantified each individual's degree as the total number of social associates. To facilitate comparison between replicate groups 1 and 2, which differed slightly in the total number of individuals in each group ( $N=21$ versus $N=19$ ), we standardized degree measures by using each individual's normalized degree, or the proportion of potential associates with which each individual associated (Freeman 1979). We summarized group-level ego network statistics by quantifying the mean normalized degree for each group during each time period and for each association type (Fig. 1d). Mean normalized degree is equivalent to network density, which is the proportion of the observed ties in the network compared to the total possible ties (Wasserman \& Faust 1994). We tested for consistency in formation patterns between replicate groups with repeated measures ANOVA (using R package ez v.3.0) to determine whether observed patterns of normalized degree differed between groups within association types.

To examine neutral association formation in more detail, we used continuous measures of SRI weighted association strengths. We quantified normalized degree strength by dividing the sum of tie strengths for each node by the maximum tie strength multiplied by the maximum number of ties. Similar to normalized degree, normalized degree strength indicates the proportion of the total possible nodal degree strength that each node achieves. We summarized these ego network measures by quantifying grouplevel ego network statistics as the mean of ego network measures across all individuals in each group for each association type at each time period.

\section{Stabilization of Global Social Structure}

To evaluate stabilization patterns of social structure, we constructed square matrices for each type of continuous measure of dyadic association strength. A separate matrix was constructed for neutral, affiliative and agonistic association types for each group and for each of the eight time periods. Each matrix contained association strength measurements quantifying how all individuals interacted with all other individuals during that time period (Fig. 1e). We used matrix permutation tests to determine the strength and statistical significance of correlations among these matrices. This method allows the use of relationship strengths and incorporates social network structure by testing for global matrix patterns using all dyadic relationship strengths in a single test. This method also avoids problems with nonindependence of data that are inherent to social network approaches (Croft et al. 2008, 
2011). The matrix approach is also appropriate for all three association types, including agonistic associations, as it depends on the score of aggression between individuals, rather than a grouplevel ranking like dominance rank. We used the quadratic assignment procedure (QAP) correlation test, which is functionally equivalent to a Mantel matrix permutation test (Legendre \& Fortin 2010). The QAP correlation test permutes the rows and columns of the observed matrix and recalculates the matrix-level correlation, then compares correlations from randomly permuted matrices to the observed matrix to determine whether observed correlations are significantly higher than those from the permuted matrices (Hubert et al. 1981; Krackhardt 1988; Borgatti et al. 2002; Croft et al. 2011). We conducted QAP correlations with 5000 permutations using the program UCINET v.6.318 (Analytic Technologies, Harvard, MA, U.S.A.).

To evaluate sequential changes in stability, we calculated correlation values between matrices of association strength for adjacent time periods, or lag 1 comparisons, and graphed these over time (Fig. 1f). We defined stable social structure as two matrices that were statistically correlated and had high correlation strength, with higher correlations taken as stronger evidence of stability between sequential time periods. By this definition, perfectly stable social structure would occur if two matrices were correlated with $P<0.05$ and $r=1.0$. In addition, to assess the statistical significance of the lag 1 correlation patterns, we ran a simulation to test the likelihood of patterns of observed lag 1 correlation strengths compared to randomized data matrices. The simulation was constructed following a model-independent test for short time series (Golinski \& Boecklen 2006). This simulation tests for nonrandom structure in lag 1 correlation strengths by permuting existing matrices, and it is an exact test, because it returns all potential combinations of the data.

Changes in network structure between sequential time periods could be incremental but result in a large amount of total change when comparing across multiple timescales. To evaluate broader stability patterns, we constructed networks that displayed correlation strengths among all time periods over the course of the entire study period (Fig. 1g). To assess consistency in formation patterns, we compared the matrices of correlation strengths among all time periods of group 1 against group 2 by association type using QAP correlation tests run for 5000 permutations.

\section{RESULTS}

\section{General Observations}

We collected more than 50000 observations of monk parakeet associations and interactions over the course of 323 hours of observer effort. Flock size ranged from 1 to 20 in group 1 $(N=10117)$ and from 1 to 19 in group $2(N=10333)$. Flocks composed of all available individuals within groups accounted for a small percentage of total flock observations (group $1=0 \%$; group $2=0.5 \%$ ). Mean \pm SD flock size was $3.24 \pm 2.80$ for group 1 and $4.24 \pm 3.92$ for group 2. Mean flock size for our captive parakeets was similar to mean foraging flock size in a feral population (4.8 birds; South \& Pruett-Jones 2000) and mean flying flock size in native populations (2.98 birds; Eberhard 1998).

Although neither group developed a consistently linear dominance hierarchy within time periods (E. Hobson, unpublished data), it was relatively easy for observers to distinguish top- and bottomranked individuals. These patterns were obvious within the first 2 days of observation for group 1. The two top-ranked individuals were a pair that had been traditionally housed together and were both randomly selected for participation in group 1 ; these individuals were rarely challenged by others and quickly monopolized a preferred perching area within the flight pen. Primary associate preferences in group 1 established quickly and remained unchanged until near the end of the 24-day study period, when two pairs suddenly separated, driving social upheaval in the group during time periods 7 and 8 . In group 2 , the top-ranked individual (a male) was also obvious to observers within the first 2 days of observation. This individual had been separated from a long-term mate due to random group assignment. During the first 7 days of observation, up to five females competed aggressively with each other to bond with this male. Unlike group 1, group 2 was characterized by a longer lag before primary social associate preferences stabilized during time periods 3 and 4 . Once primary associate preference formed, group 2 did not undergo a social upheaval such as observed in group 1 and associate preferences remained stable through the end of the study period for group 2 .

Randomization tests of neutral associations provide evidence that flocking association patterns were nonrandom. Observed mean association strength, standard deviation of association strength and the coefficient of variation all differed from expected values at all time periods and for both replicate groups $(P \geq 0.9999$ in all cases).

\section{Formation of Dyadic Associations}

Random assignment of individuals into the two replicate groups resulted in the formation of many novel dyadic associations; $97 \%$ of group 1 and $94 \%$ of group 2 dyads were associations between individuals not housed together within 8 months preceding the study. While some dyadic associations showed clear patterns of strengthening or weakening over time, association trajectories were generally poorly described by regressions of association strength on time (Table 1). Neutral associations showed the highest percentage of significant dyad trajectories. Of the dyads with statistically significant trajectories, almost all were trajectories with positive slopes, indicating that neutral associations generally

Table 1

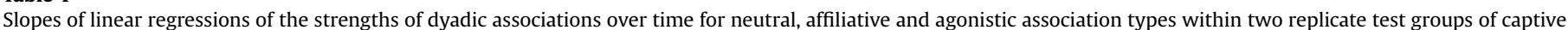
monk parakeets (as in Fig. 1d)

\begin{tabular}{|c|c|c|c|c|c|c|}
\hline \multirow[t]{2}{*}{ Group } & \multirow[t]{2}{*}{ Association type } & \multirow[t]{2}{*}{ Mean slope \pm SD } & \multicolumn{3}{|l|}{ \% Significant* } & \multirow[t]{2}{*}{ Mean $\mathrm{MSE} \pm \mathrm{SD}$} \\
\hline & & & Trajectories & Positive slopes & Negative slopes & \\
\hline \multirow[t]{3}{*}{ Group 1} & Neutral & $0.026 \pm 0.02$ & 32.86 & 98.55 & 1.45 & $0.009 \pm 0.01$ \\
\hline & Affiliative & $<0.001 \pm 0.05$ & 9.05 & 36.84 & 63.16 & $0.058 \pm 0.20$ \\
\hline & Agonistic & $0.021 \pm 0.03$ & 8.33 & 85.71 & 14.29 & $0.031 \pm 0.02$ \\
\hline \multirow[t]{3}{*}{ Group 2} & Neutral & $0.029 \pm 0.02$ & 36.84 & 100.00 & 0.00 & $0.009 \pm 0.01$ \\
\hline & Affiliative & $<0.001 \pm 0.07$ & 8.19 & 57.14 & 42.86 & $0.064 \pm 0.21$ \\
\hline & Agonistic & $<0.001 \pm 0.03$ & 9.36 & 50.00 & 50.00 & $0.021 \pm 0.01$ \\
\hline
\end{tabular}

MSE: mean square error.

* Alpha $=0.05$. 
strengthened over time. Only one dyad in group 1 had a neutral association that significantly weakened over time. In contrast, fewer affiliative and agonistic association trajectories were described by the regressions. For affiliative associations, group 1 had more associations that weakened over time than strengthened. In contrast, group 2 had more affiliative associations that strengthened over time than those that weakened.

Association trajectories in all groups and across all association types generally had very small slope values (Table 1 ). When we examined variation of residuals around regression lines, we found that neutral association trajectories had the lowest average MSE by group, followed by agonistic associations (Table 1 ). Of the three association types, affiliative association trajectories had the highest average MSE and were least well described by linear relationships.

When we examined residual size for insight into times when association strength deviated most from the linear relationship, we found that association trajectories exhibited qualitatively similar patterns of the timing and extent of residual size (deviation from the fitted linear relationship; Fig. 2). All three association types exhibited largest mean residual sizes in time period 1 across both replicate social groups. Neutral associations consistently showed the smallest mean residual size for both groups at all time periods, but affiliative and agonistic mean residual size showed different patterns between group 1 and group 2, with group 1's affiliative association mean residual size increasing at time period 8, corresponding to observed social upheaval and shifts in dyadic associations at that time.

\section{Formation of Ego Network Structure}

Both replicate social groups showed qualitatively similar patterns in the formation of local social structure (Fig. 3). Individuals had the highest number of neutral social associates, a moderate number of affiliative associates and the least number of agonistic associates in all time periods. This resulted in the highest network connectivity for neutral associations, measured through shared flock membership, and the lowest connectivity for agonistic associations, measured through directed aggression (Fig. 4).

Unfiltered neutral association networks were perfectly connected for both groups, with all individuals scoring maximum normalized degree (1.0), indicating that all individuals were sighted at least once in the same flock with all other individuals during each time period. Because this perfect connectivity could be due to rare observations of dyadic associations, we filtered our network so that only dyads observed associating in more than $5 \%$ of sampling periods during any one time period were included. Even with this filtering of associations, mean normalized degree for neutral associations remained very high with most time periods showing perfect network connectivity (Supplementary Material, Fig. S1). Because filtered networks differed little from unfiltered networks, it is likely that the high connectivity of neutral networks is biologically relevant, rather than an artefact of rare occurrences of associations. To evaluate patterns of neutral network formation in more detail, we also considered temporal dynamics in normalized degree strength using the continuous weighted dyadic association strengths. We found that degree strength in both groups increased over time (Fig. 5). This result, in combination with the perfectly connected binary neutral networks, shows that although the total numbers of ties did not change, the strength of ego network ties largely increased over time.

In contrast to the very high (filtered networks) to perfect (unfiltered networks) connectivity of neutral networks, aggressive interaction networks and affiliative association networks showed overall lower connectivity, with individuals interacting with a subset of all potential associates during each time period. Aggressive networks were the least connected of the three association types, with the lowest average number of associates for each time period.

We tested for consistency in social structure formation between groups 1 and 2 using a repeated measures ANOVA on normalized degree over time and found mixed results. In all cases, Mauchly's test of sphericity indicated that sphericity could not be assumed (neutral: $P<0.0001$; affiliative: $P<0.0001$; agonistic: $P=0.0159$ ) so we used the Greenhouse-Geisser correction for nonsphericity in subsequent tests. Unfiltered binary neutral associations were perfectly connected at all time periods with zero variance and, thus, there was no difference between groups 1 and 2, showing that groups did not differ in patterns of neutral association formation. In contrast, patterns of normalized degree strength for neutral associations differed significantly between groups. We found a significant relationship with time, group, and group by time $(P<0.0001$ in all cases). Degree formation patterns also differed significantly between groups for both affiliative and agonistic association types. We found a significant relationship with time (affiliative: $P<0.0001$; agonistic: $P<0.0001$ ), group (affiliative: $P<0.0001$; agonistic: $P<0.001$ ), and group by time (affiliative: $P<0.0001$; agonistic: $P<0.0001)$. These results indicate that while binary neutral social structure formed in similar ways for both groups 1

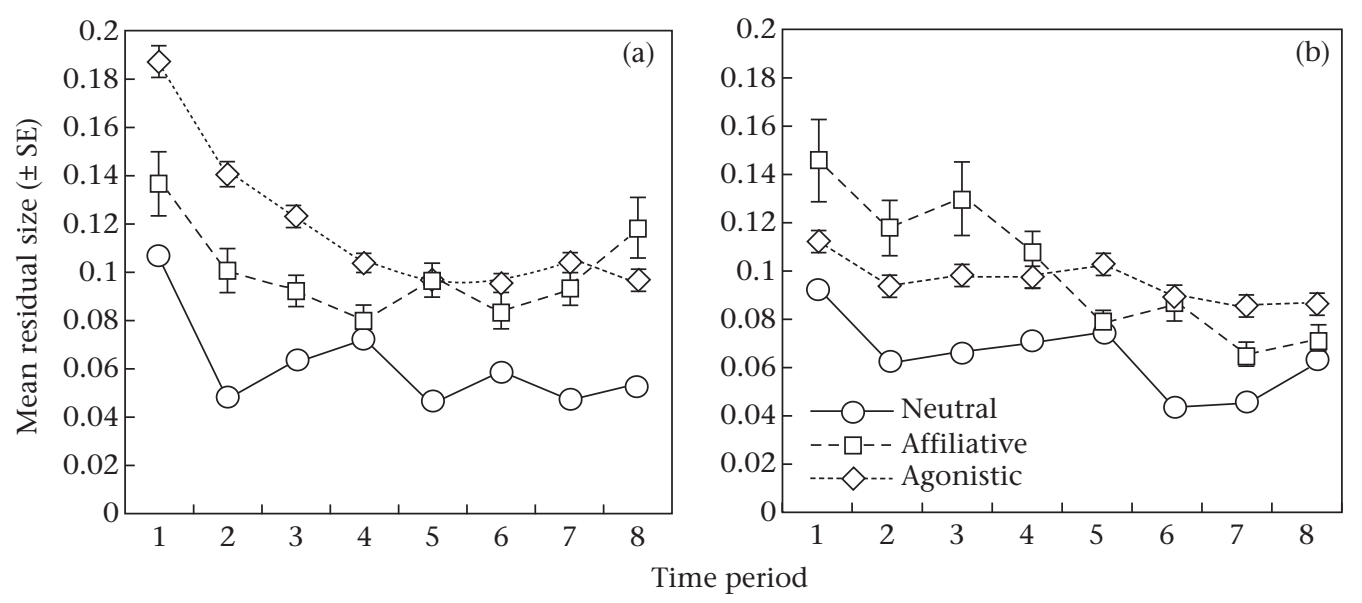

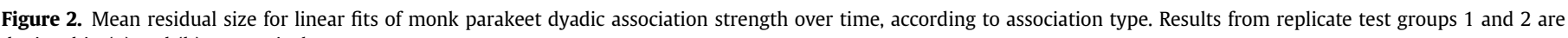
depicted in (a) and (b), respectively. 


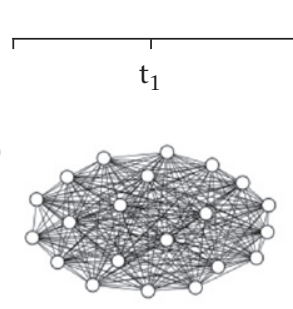

(b)

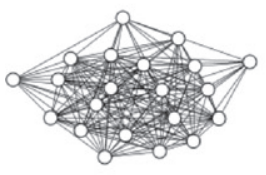

(c)

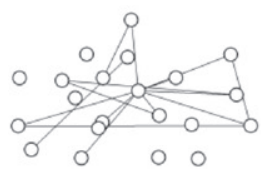

Group1

$t_{4}$
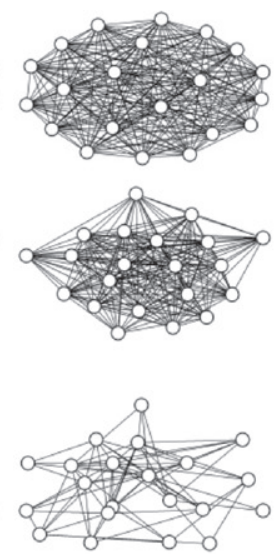

$t_{8}$
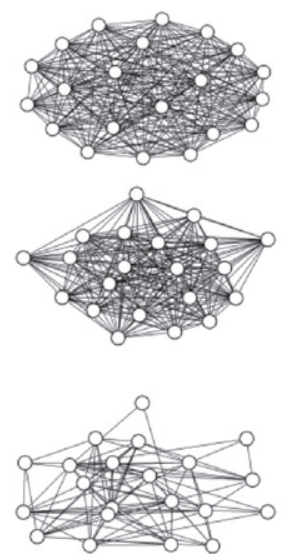

Group2

\begin{tabular}{lll}
\hline$t_{1}$ & $t_{4}$ & $t_{8}$
\end{tabular}
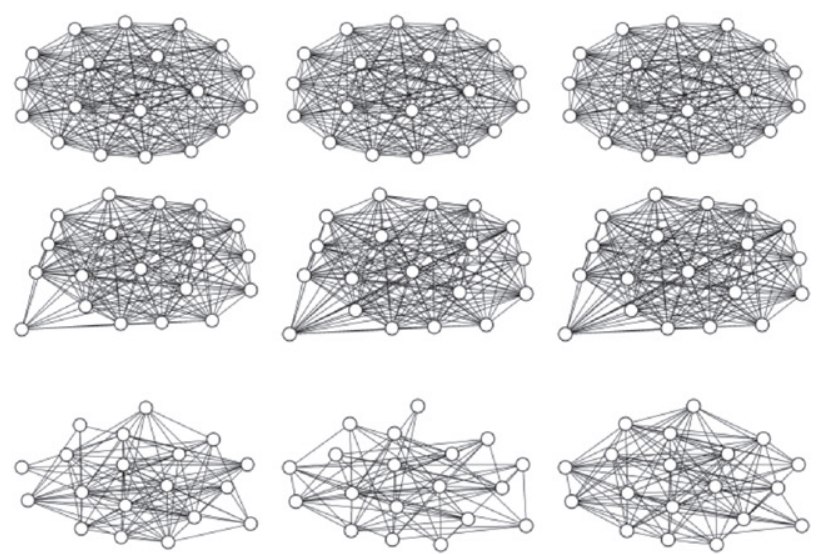

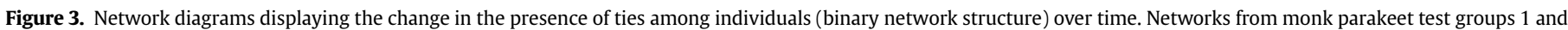

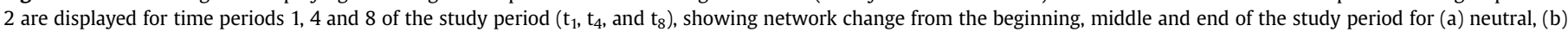

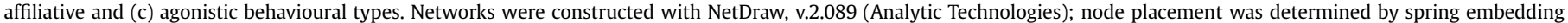
algorithm.

and 2, formation patterns differed between groups for weighted measures of neutral social structure and binary measures of affiliative and agonistic structures.

\section{Stabilization of Global Social Structure}

Sequential stabilization patterns, evaluated through lag 1 correlation strengths between networks, varied widely (range 0.034-0.976; Fig. 6). However, all sequential correlations exhibited enough similarity in networks to return statistically significant correlations $(P<0.05)$ between time periods. Correlation strengths were lowest across both groups and all association types when comparing matrix structure from time 1 to time 2, indicating that the greatest sequential change in social associations occurred between these time periods. When considered across all time periods, all lag 1 correlations showed similar asymptotic patterns, as correlation strengths increased over time and then levelled off. Both affiliative and neutral structures quickly increased to high correlation strengths, reached higher correlation values than agonistic structure, and thus displayed stronger evidence for stable social structure. Affiliative structure showed the most evidence for stabilization, with almost perfect stability in both groups during the middle of the study period. Contrary to our prediction, agonistic structure formed most slowly in each group and had the lowest correlation strengths between sequential matrices, demonstrating less evidence for stable social structure. Computer simulation tests determined the statistical differences between observed lag 1 correlation values and those expected from all combinations of
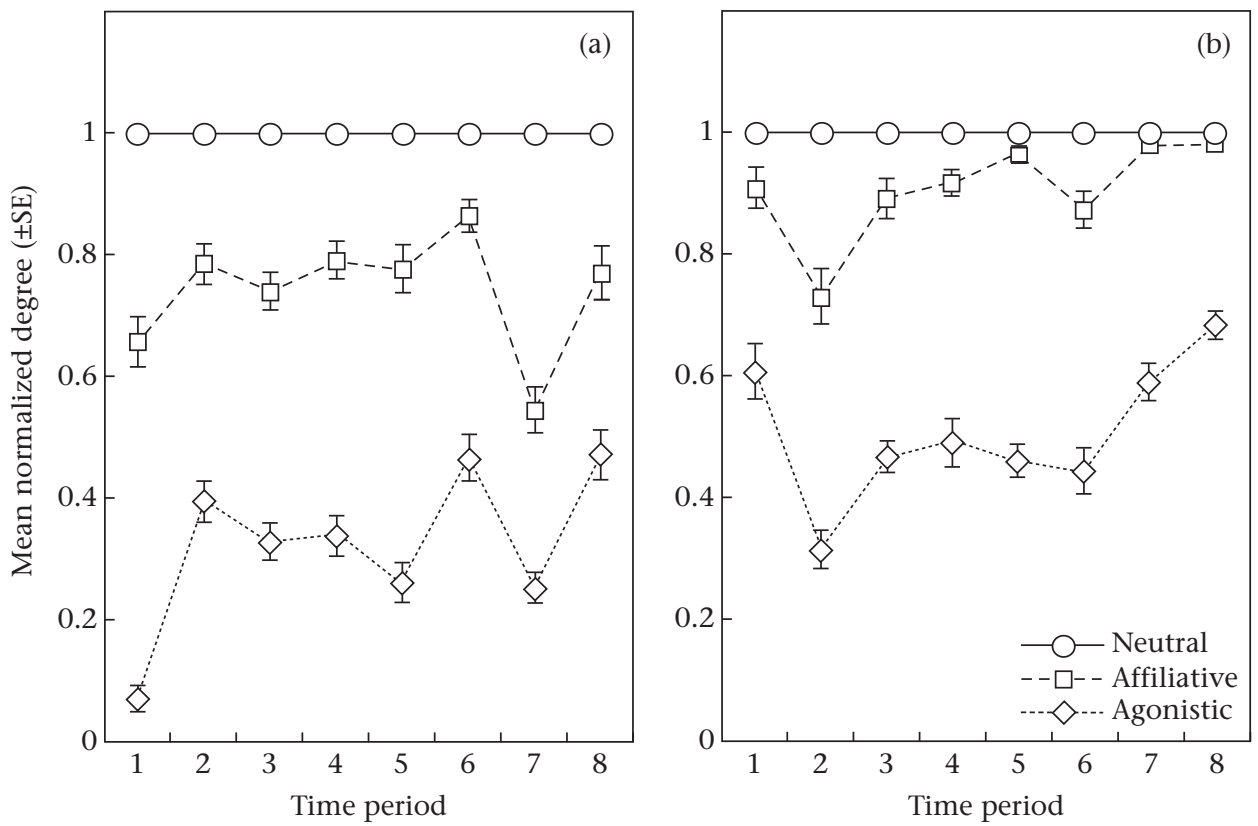

Figure 4. Patterns of change in ego network structure (as in Fig. 1d). Graphs display mean normalized degree for group 1 (a) and group 2 (b) by association type over time. 


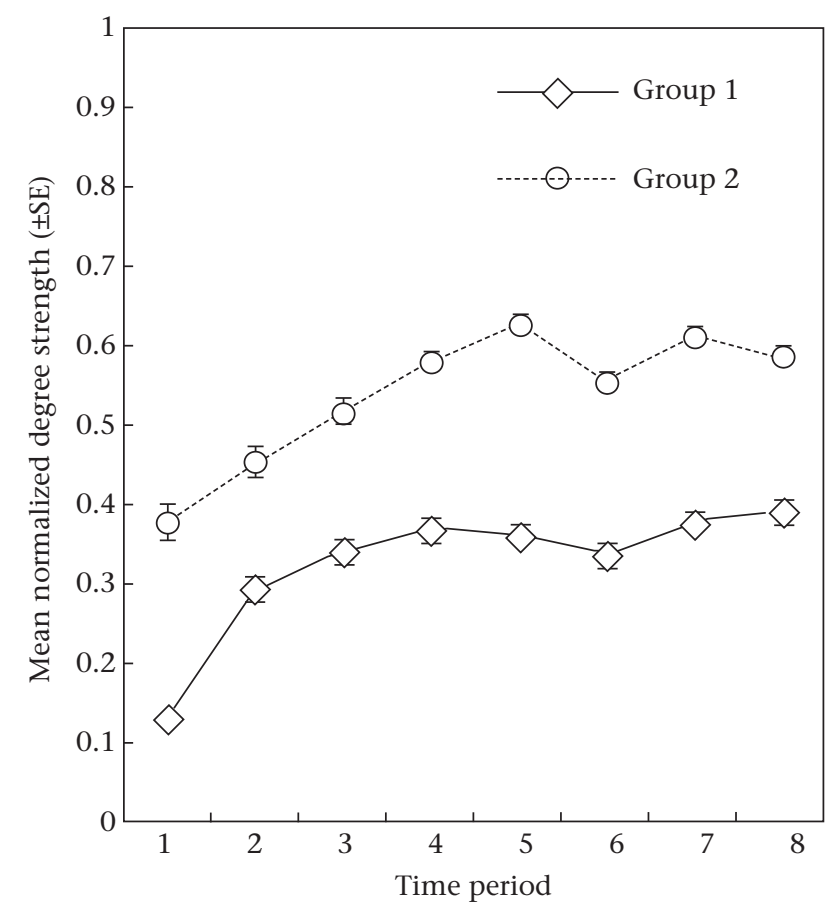

Figure 5. Mean normalized degree strength over time for neutral associations in both monk parakeet test groups. Although patterns were qualitatively similar, groups differed statistically in formation patterns.

permuted data. Lag 1 correlations showed evidence for nonrandom structure in both groups for all association types except for group 1 agonistic structure (Table 2).

Broader stabilization patterns were evaluated through correlating all matrices for all time periods over the entire study period. QAP matrix permutation tests showed that correlations among almost all time periods were statistically significant but varied in correlation strength. Correlation networks showed strong correlations and high mean intercorrelation values across association types after time period 1 or 2 , implying that stable social structure formed relatively quickly and remained stable across multiple time
Table 2

Results of a computer simulation test to assess whether observed lag 1 correlation strengths differed from all possible lag 1 correlations of randomly permuted matrices

\begin{tabular}{lll}
\hline Association type & Pearson $r(P)$ & \\
\cline { 2 - 3 } & Group 1 & Group 2 \\
\hline Neutral & $0.776(<\mathbf{0 . 0 0 1})$ & $0.705(\mathbf{0 . 0 0 7})$ \\
Affiliative & $0.880(\mathbf{0 . 0 0 3})$ & $0.874(\mathbf{0 . 0 1 5})$ \\
Agonistic & $0.371(0.295)$ & $0.551(\mathbf{0 . 0 4 5})$ \\
\hline
\end{tabular}

Significant $P$ values $(P<0.05)$ are shown in bold.

Evidence of nonrandom structure occurred for each comparison except agonistic associations in group 1 .

lag lengths (Fig. 7). Overall, average correlation strength varied among social association types, with affiliative associations showing the highest average correlation strength. We found a significant correlation between the formation patterns of groups 1 and 2 in all cases except for lag 1 neutral association (Table 3 ). These results provide evidence for generally consistent patterns for social structure formation across the two replicate groups.

\section{DISCUSSION}

In this study, we described a framework for analysis of the temporal dynamics of social structure that integrates observations of social associations across social scales and association types. We used this analytical framework to visualize, quantify and test social network formation and stabilization patterns in captive groups of monk parakeets. Results from quantitative analyses within this framework corresponded to our qualitative observations of social structure dynamics. Social structure formed and stabilized over a short period in captive groups of monk parakeets, but the details of these temporal patterns differed by social association type. We found general evidence for consistency in the temporal dynamics of formation and stabilization of social structure between replicate social groups. We discuss the generalizability of this framework to diverse taxa with different underlying social structures across widely varying timescales, and we evaluate its usefulness in providing insight into the characterization of the importance of social structure.
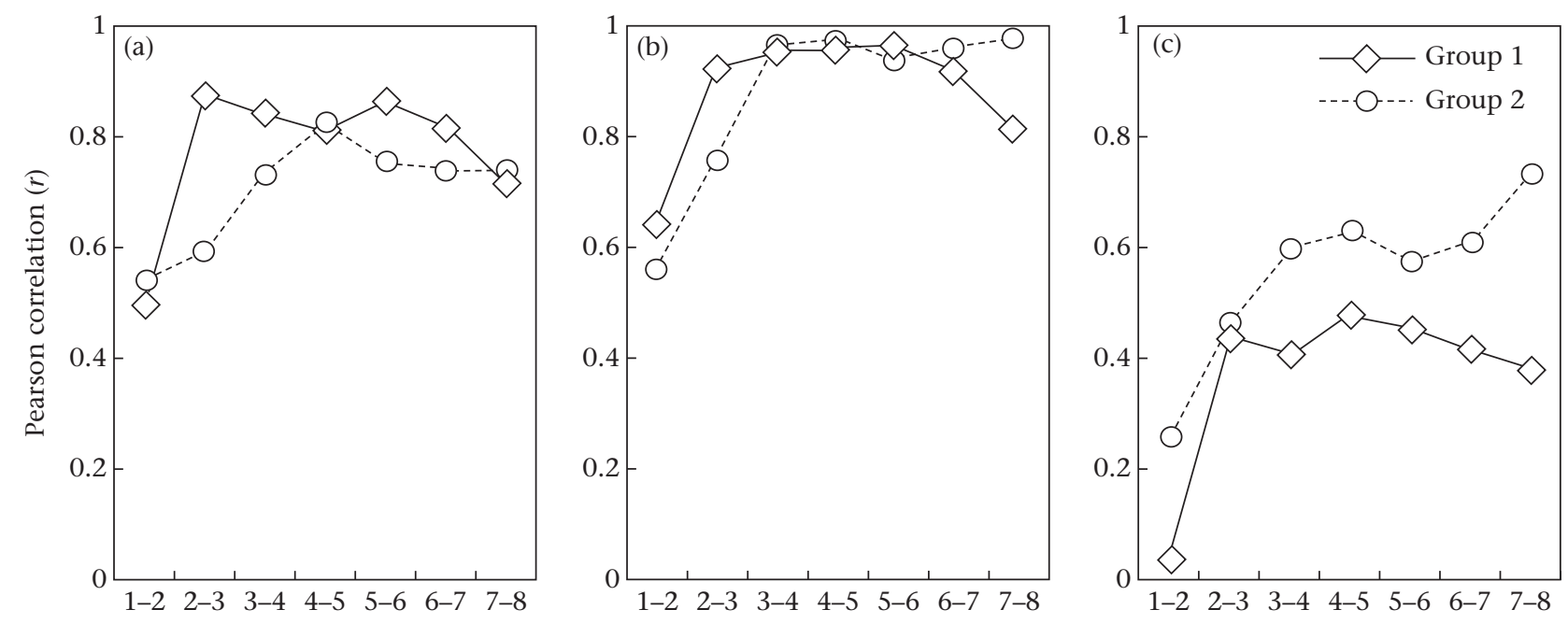

Time period for matrix comparison

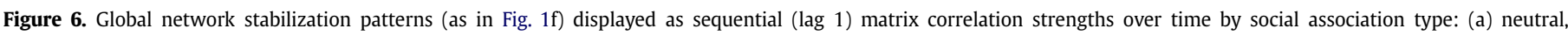
(b) affiliative, (c) agonistic. Lag 1 correlation strengths from quadratic assignment procedure (QAP) correlation tests were all statistically significant at $P<0.05$. 

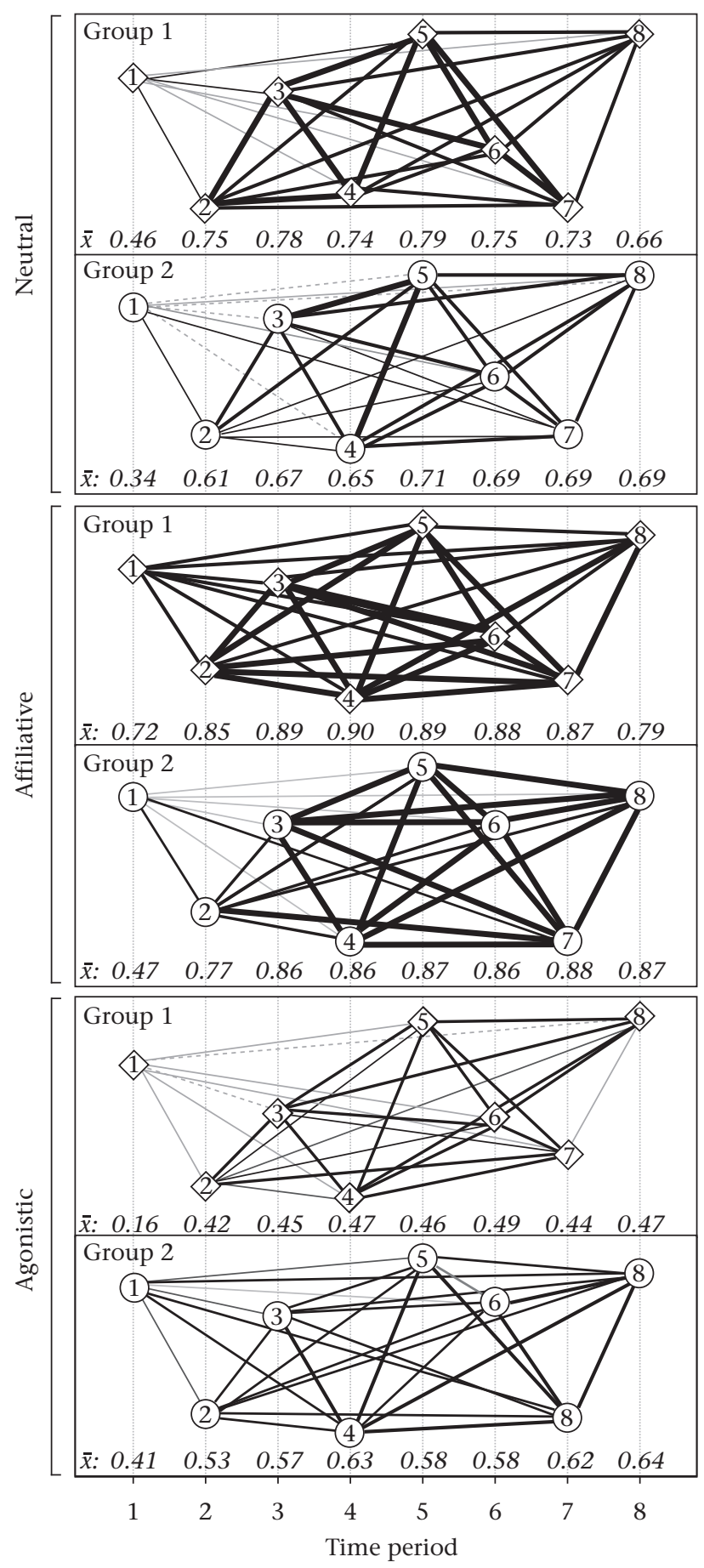

\begin{tabular}{|l|} 
Correlation strength $(r)$ \\
$r<0.2, P>0.05 \quad-0.4 \leq r<0.6$ \\
$r<0.2, P \leq 0.05 \quad-0.6 \leq r<0.8$ \\
$0.2 \leq r<0.4 \quad-r \geq 0.8$
\end{tabular}

Figure 7. Global network stabilization patterns (as in Fig. 1g) displayed as correlation strengths among time periods throughout the study. Nodes correspond to each of the eight time periods; the widths of ties among time periods depict Pearson correlation strength $(r)$. All correlations among time periods were statistically significant at $P<0.05$ except those depicted by dashed lines. Mean intercorrelation values for each time period are shown in italics at the bottom of each diagram.
Table 3

Consistency in formation of social structure between groups for neutral, affiliative and agonistic association types evaluated using Pearson correlations between group 1 and group 2

\begin{tabular}{lll}
\hline Association type & Pearson $r(P)$ & \\
\cline { 2 - 3 } & Lag 1 & All lags \\
\hline Neutral & $0.57(0.1847)$ & $0.76(\mathbf{0 . 0 4 4 6 )}$ \\
Affiliative & $0.76(\mathbf{0 . 0 4 5 7})$ & $0.73(\mathbf{0 . 0 3 7 8})$ \\
Agonistic & $0.78(\mathbf{0 . 0 3 7 5 )}$ & $0.84(\mathbf{0 . 0 2 0 8 )}$ \\
\hline
\end{tabular}

Significant $P$ values $(P<0.05)$ are shown in bold.

Formation and Stabilization of Social Structure in Monk Parakeets

We found that aspects of monk parakeet social structure could form and stabilize in a short period. At the dyadic level, most pairwise associations had small slope values and showed no clear pattern of strengthening or weakening through time. Analysis of residual size indicated a relatively good fit with a linear relationship. This implies that even with small slopes, dyadic associations can be characterized as linear relationships and that those associations are generally stable on the dyadic scale.

At the individual or ego network scale, we found that monk parakeet social network formation patterns were qualitatively consistent across replicate social groups. In both groups, individuals had the highest number of neutral social associates, a moderate number of affiliative associates and the least number of agonistic associates at all time periods. Neutral associations showed consistent formation patterns between groups. Although general patterns of affiliative and agonistic association formation were qualitatively consistent between groups, groups differed significantly in formation patterns for these association types. Differences in degree through time between groups were most likely due to differences in how groups interacted during the study period: higher degree during time period 1 in group 2 for affiliative and agonistic associations were probably due to the intense competition among females vying for one particular male. Group 1 showed a drop in normalized degree at time period 7 for both affiliative and agonistic associations, corresponding to observations of pair rupture and reformation and suggesting that social contacts decreased during this abrupt shift in social structure.

At the global network scale, we found nonrandom structure in matrix correlations that provides evidence of stabilization in monk parakeet social structure. Affiliative, agonistic and neutral associations all showed evidence of both incremental and broader-scale stability. For both groups 1 and 2, affiliative structure showed the strongest evidence for stability, while evidence for stability was weakest in agonistic associations. Both sequential and broaderscale analysis methods detected decreased similarity in social structure during the end of the study period when group 1 associations underwent several changes: lag 1 correlation strengths decreased when comparing time periods 7 and 8 , and time 8 showed lower mean correlation strengths. We also found that patterns of sequential and broader social structure stabilization were both consistent between replicate social groups in almost all categories.

While it is possible that established dyadic associations could cause more rapid stabilization of social structure, established dyads in our case accounted for a small proportion of the total dyads. Because matrix permutation methods considered all dyadic association strengths, it is unlikely that the few established associations were responsible for driving observed stabilization patterns. Furthermore, the effect of previous associations should be distributed equally across all three association types. Additionally, because there were similar proportions of established and novel 
associations in groups 1 and 2, the effect of previous associations should also equally affect both replicate groups.

Quantification of formation and stabilization patterns of social structure can provide insight into the relative importance of different types of social structure. Contrary to predictions, monk parakeets formed agonistic associations more slowly than affiliative or neutral associations and showed weaker evidence for network stabilization during the study period. Because of nonindependence in our data sets, we were unable to formally test for statistical differences among association types within replicate social groups. However, the consistent patterns of formation and stabilization between replicate social groups suggest that agonistic associations are the least stable of the association types considered in this study. The difference in temporal dynamics of different types of social structures may provide an indication that affiliative associations are more important than agonistic associations in monk parakeets. Individuals may benefit more from affiliative relationships, perhaps through passive food sharing, aid in nest building, or access to foraging information, than from agonistic relationships. If benefits of relationships are skewed towards affiliative relationships, it may explain why those relationships formed and stabilized more quickly than agonistic relationships. Further study of information sharing and long-term fitness correlates of social network metrics could provide additional insight into monk parakeet social structure.

Greater knowledge of monk parakeet social dynamics can also increase our understanding of how the parakeets respond to social upheaval events. Monk parakeets naturally encounter situations in which novel groups may form quickly in response to disturbance. In both the native and nonnative ranges, stochastic weather events and anthropogenic disturbances such as nest removal and eradication programmes (Tillman et al. 2004; Pruett-Jones et al. 2005; Avery et al. 2006; Burger \& Gochfeld 2009) can alter social composition and promote regrouping and formation of novel groups. Monk parakeets are also successful invasive species that have established multiple introduced populations, largely stemming from accidental escapes and intentional releases of pet birds (Russello et al. 2008). Our study conditions simulated several of the conditions expected during establishment of a novel introduced population stemming from a group release. Greater understanding of the short-term formation and stabilization dynamics of social structure could also provide insight into the social processes that occur following an introduction event and could be used to provide indications of how social behaviours impact invasion dynamics and success.

\section{Evaluation of the Analytical Framework}

The multiscale multiplex analytical framework that we utilized in this study allowed us to quantify and test patterns of social structure formation and stabilization in captive groups of monk parakeets. We examined and tested network dynamics on the dyadic, ego network and global network scales and across neutral, affiliative and agonistic social association types. Our analysis methods successfully identified change in social structure; quantitative analyses of network dynamics corresponded well with qualitative observations of social behaviour. Utilizing methods that considered multiple social scales provided insight into the dynamics of networks. For example, at the ego network scale and considering simple network connectivity or the number of social associates, networks were perfectly connected for neutral associations while affiliative networks were less connected. In contrast, neutral associations showed moderate evidence for stability at the global network scale while affiliative associations showed the most evidence. Comparing across social scales provides a more holistic perspective on dynamic network change. In addition, our multiplex approach of quantifying and testing the dynamics of social structures in three different social association types also provided additional insight. Comparison of neutral, affiliative and agonistic associations showed differences in network formation and stabilization patterns. These differences allowed us to draw inferences to the potential relative importance of the different association types to the parakeets and provided insight into factors structuring monk parakeet sociality.

Our methods are also useful for comparing across groups that differ in size. Enabling this comparison is crucial to comparative studies, but few robust methods have been available (Croft et al. 2008). Our framework provides methods to facilitate these comparisons. At the dyadic level, the focus on dyadic associations makes differences in group size a minor issue. At the ego network scale, standardization methods are available for some network metrics that can enable direct comparisons between differently sized groups. In many cases, network metrics based on binary data are easier to normalize than weighted measures ( $T$. Opsahl, personal communication). In our case, we found that degree was especially amenable to standardization and interpretation across differently sized groups. At the global network scale, we utilized methods to permute networks for each group at each time period. We could not directly compare matrices of association strengths between groups because these methods require equally sized matrices (Croft et al. 2008). Instead, we focused on the resulting within-group matrix correlation strengths to compare betweengroup patterns of network change over time directly.

The analytical framework is also valuable because it can be utilized for studying the temporal dynamics of social structure in diverse taxa, across many types of social structures, and at many different timescales. For example, a similar scaled analysis was used to study of Asian elephant, Elephas maximus, social dynamics to determine how social associations changed at the dyadic, ego network and global network scales across seasons (de Silva et al. 2011). We demonstrate the applicability of this framework for quantifying and testing patterns of formation and stabilization of social structure in a very different species, with different basic social structure, and over a different timescale. The elephant study utilized data on a seasonal timescale, with data aggregated over several months. In contrast, our work with the parakeets utilized data on a much finer timescale, with data pooled into 3-day aggregations. Our analysis methods are especially applicable to temporal analyses of data where traditional time series analysis is not possible because of lack of sufficient longitudinal data points. In addition, because this analytical framework is largely free of underlying assumptions about social structure, it can be applied to diverse taxa with very different association patterns. Our methods can be utilized across multiple social association types and in groups with fission-fusion social systems and those without strong dominance hierarchical structure. Furthermore, our methods are robust to comparison of temporal dynamics across groups of different sizes. Our methods also rely heavily on weighted measures of association strength and can be used with weighted directed networks where associations are asymmetric. Generally, the study questions, the type of data and the characteristics of the study species will drive the choice of specific methods for quantifying and testing temporal dynamics. However, the overall scaled social analysis framework is broadly generalizable to a wide variety of species, social structures and timescales.

One drawback to analyses in our framework is that they are sensitive to situations where individuals join and leave social groups. In particular, the stability analyses and matrix correlation methods require that all matrices be same size with the same individuals represented in each. In groups with high membership turnover, 
these methods are likely to be less applicable. Our methods require either relatively stable group membership or analysis on a short enough time span that gross group membership is not altered by emigration, immigration, birth or deaths. In addition, comparisons can be made among independent replicate social groups, but require data to be samples on a similar time span; in our case, this was accomplished by pooling observation days into eight 3-day bins.

Finally, this analytical framework can determine temporal changes in social structure, characterize patterns of network dynamics, quantify network change and test network formation and stabilization patterns. However, the analyses used in this study do not address why associations and structures change, nor do they identify factors that drive changes in sociality. Stochastic actorbased models can be used to model network dynamics driven by multiple tendencies or factors and used to predict the creation or dissolution of ties within networks (Snijders et al. 2010).

\section{Conclusion}

Our study methods are likely to be useful in characterizing patterns of temporal dynamics in social structure in longitudinal data in a wide variety of social systems and species. Implementation of these methods for visualizing, quantifying and testing patterns of change in social networks will increase our understanding of how social structure changes over time. Detailed information on the dynamics of social structure across multiple association types can also provide insight into the general characterization of social structure and can aid in predicting the costs and benefits of individual social investment strategies. Increased understanding of social network dynamics can then allow studies to move beyond description of patterns to prediction and theories for explaining the ultimate causes of structural change.

\section{Acknowledgments}

We thank our field technicians, Darlene John, Tiffany McIntosh and Aaron Hobson, who were indispensable to data collection, the staff of the Florida Field Station for their hospitality and support, Kandy Keacher for general animal care and aviary record maintenance, Tore Opsahl for advice on network metric normalization and R, Marcelo Araya Salas for R scripts, and Bill Boecklen for computer model construction and statistical advice. Comments from B. Boecklen, A. Salinas-Melgoza, M. Araya-Salas, B. Ryder, an anonymous referee, and attendants at the 2012 Symposium on Network Science in Biological, Social, and Geographic Systems greatly improved this manuscript. Funding was provided by the American Ornithologists' Union, Sigma Xi and National Science Foundation (NSF) GK-12 Program (number DGE-0947465) to E.H. and NSF number IOS-0725032 to T.W.

\section{Supplementary Material}

Supplementary material for this article is available, in the online version, at http://dx.doi.org/10.1016/j.anbehav.2012.10.010.

\section{References}

Anderson, C. J., Wasserman, S. \& Crouch, B. 1999. A p* primer: logit models for social networks. Social Networks, 21, 37-66.

Avery, M. L., Lindsay, J. R., Newman, J. R., Pruett-Jones, S. \& Tillman, E. A. 2006 Reducing monk parakeet impacts to electric utility facilities in south Florida. Advances in Vertebrate Pest Management, 4, 125-136.

Barthélemy, M., Barrat, A., Pastor-Satorras, R. \& Vespignani, A. 2005. Characterization and modeling of weighted networks. Physica A: Statistical Mechanics and its Applications, 346, 34-43.

Bejder, L., Fletcher, D. \& Bräger, S. 1998. A method for testing association patterns of social animals. Animal Behaviour, 56, 719-725.
Blonder, B. \& Dornhaus, A. 2011. Time-ordered networks reveal limitations to information flow in ant colonies. PLoS One, 6, 1-8.

Borgatti, S. 2010. Simple Models for Analyzing Network Change (Webcast 53). Detroit, Michigan: Wayne State University, Center for the Advancement of Research Methods and Analysis (CARMA). http://carma.wayne.edu/VideoLibrary.asp.

Borgatti, S. P., Everett, M. G. \& Freeman, L. C. 2002. UCINET for Windows: Software for Social Network Analysis. Version 6.318. Harvard, Massachusetts: Analytic Technologies.

Buckley, N. J. 1996. Food finding and the influence of information, local enhancement, and communal roosting on foraging success of North American vultures. Auk, 113, 473-488.

Burger, J. \& Gochfeld, M. 2009. Exotic monk parakeets (Myiopsitta monachus) in New Jersey: nest site selection, rebuilding following removal, and their urban wildlife appeal. Urban Ecosystems, 12, 185-196.

Cairns, S. J. \& Schwager, S. J. 1987. A comparison of association indices. Animal Behaviour, 35, 1454-1469.

Chase, I. D. 1980. Social process and hierarchy formation in small groups: a comparative perspective. American Sociological Review, 45, 905-924.

Chase, I. D. 1982. Dynamics of hierarchy formation: the sequential development of dominance relationships. Behaviour, 80, 218-240.

Chase, I. D., Tovey, C., Spangler-Martin, D. \& Manfredonia, M. 2002. Individual differences versus social dynamics in the formation of animal dominance hierarchies. Proceedings of the National Academy of Sciences, U.S.A., 99, 5744-5749.

Croft, D. P., James, R. \& Krause, J. 2008. Exploring Animal Social Networks. Princeton, New Jersey: Princeton University Press.

Croft, D. P., Madden, J. R., Franks, D. W. \& James, R. 2011. Hypothesis testing in animal social networks. Trends in Ecology \& Evolution, 26, 502-507.

Drews, C. 1993. The concept and definition of dominance in animal behaviour. Behaviour, 125, 283-313.

East, M. L., Honer, O. P., Wachter, B., Wilhelm, K., Burke, T. \& Hofer, H. 2009. Maternal effects on offspring social status in spotted hyenas. Behavioral Ecology, 20, 478-483.

Eberhard, J. R. 1998. Breeding biology of the monk parakeet. Wilson Bulletin, 110, 463-473.

Fischhoff, I. R., Sundaresan, S. R., Cordingley, J., Larkin, H. M., Sellier, M.-J. \& Rubenstein, D. I. 2007. Social relationships and reproductive state influence leadership roles in movements of plains zebra, Equus burchelli. Animal Behaviour, 73, 825-831.

Foster, E. A., Franks, D. W., Morrell, L. J., Balcomb, K. C., Parsons, K. M., van Ginneken, A. \& Croft, D. P. 2012. Social network correlates of food availability in an endangered population of killer whales, Orcinus orca. Animal Behaviour, 83, 731-736.

Freeman, L. C. 1979. Centrality in social networks conceptual clarification. Social Networks, 1, 215-239.

Ginsberg, J. R. \& Young, T. P. 1992. Measuring association between individuals or groups in behavioural studies. Animal Behaviour, 44, 377-379.

Golinski, M. \& Boecklen, W. J. 2006. A model-independent test for the presence of regulatory equilibrium and non-random structure in island species trajectories. Journal of Biogeography, 33, 1566-1570.

Hamede, R. K., Bashford, J., McCallum, H. \& Jones, M. 2009. Contact networks in a wild Tasmanian devil (Sarcophilus harrisii) population: using social network analysis to reveal seasonal variability in social behaviour and its implications for transmission of devil facial tumour disease. Ecology Letters, 12, 1147-1157.

Hamill, J. T. 2006. Analysis of layered social networks. Ph.D. thesis, Air Force Institute of Technology, Wright-Patterson AFB, OH, U.S.A

Henzi, S., Lusseau, D., Weingrill, T., van Schaik, C. \& Barrett, L. 2009. Cyclicity in the structure of female baboon social networks. Behavioral Ecology and Sociobiology, 63, 1015-1021.

Herberholz, J., Sen, M. M. \& Edwards, D. H. 2003. Parallel changes in agonistic and non-agonistic behaviors during dominance hierarchy formation in crayfish. Journal of Comparative Physiology A: Neuroethology, Sensory, Neural, and Behavioral Physiology, 189, 321-325.

Hinde, R. A. 1976a. Interactions, relationships and social structure. Man, 11, 17.

Hinde, R. A. 1976b. On describing relationships. Journal of Child Psychology and Psychiatry, 17, 1-19.

Holekamp, K. E. \& Smale, L. 1991. Dominance acquisition during mammalian social development: the 'inheritance' of maternal rank. American Zoologist, 31, 306-317.

Hubert, L. J., Golledge, R. G. \& Costanzo, C. M. 1981. Generalized procedures for evaluating spatial autocorrelation. Geographical Analysis, 13, 224-233.

Krackhardt, D. 1988. Predicting with networks: nonparametric multiple regression analysis of dyadic data. Social Networks, 10, 359-381.

Krause, J. \& Ruxton, G. 2010. Important topics in group living. In: Social Behaviour: Genes, Ecology, and Evolution (Ed. by T. Székely, A. J. Moore \& J. Komdeur), pp. 203-225. Cambridge: Cambridge University Press.

Krebs, J. R. \& Davies, N. B. 1996. An Introduction to Behavioural Ecology. Oxford: Blackwell Science.

Legendre, P. \& Fortin, M.-J. 2010. Comparison of the Mantel test and alternative approaches for detecting complex multivariate relationships in the spatial analysis of genetic data. Molecular Ecology Resources, 10, 831-844.

Lusseau, D., Wilson, B., Hammond, P. S., Grellier, K., Durban, J., Parsons, K. M., Barton, T. R. \& Thompson, P. M. 2006. Quantifying the influence of sociality on population structure in bottlenose dolphins. Journal of Animal Ecology, 75, $14-24$. 
Lusseau, D., Whitehead, H. \& Gero, S. 2008. Incorporating uncertainty into the study of animal social networks. Animal Behaviour, 75, 1809-1815.

McDonald, D. B. 2007. Predicting fate from early connectivity in a social network Proceedings of the National Academy of Sciences, U.S.A., 104, 10910-10914.

Manly, B. F. J. 1995. A note on the analysis of species co-occurrences. Ecology, 76, 1109-1115.

Martín, L. F. \& Bucher, E. H. 1993. Natal dispersal and first breeding age in monk parakeets. Auk, 110, 930-933.

Mitani, J. C. 2009. Male chimpanzees form enduring and equitable social bonds. Animal Behaviour, 77, 633-640.

Mucha, P. J., Richardson, T., Macon, K., Porter, M. A. \& Onnela, J.-P. 2010. Community structure in time-dependent, multiscale, and multiplex networks. Science, 328, 876-878.

Oh, K. P. \& Badyaev, A. V. 2010. Structure of social networks in a passerine bird: consequences for sexual selection and the evolution of mating strategies. American Naturalist, 176, E80-E89.

Opsahl, T. 2009. Structure and Evolution of weighted networks. Ph.D. thesis, University of London.

Opsahl, T. 2011. $R$ Package 'tnet'. Version 3.0.5. Vienna, Austria: Comprehensive R Archive Network, R Foundation for Statistical Computing

Patriquin, K., Leonard, M., Broders, H. \& Garroway, C. 2010. Do social networks of female northern long-eared bats vary with reproductive period and age? Behavioral Ecology and Sociobiology, 64, 899-913.

Pruett-Jones, S., Newman, J. R., Newman, C. M. \& Lindsay, J. R. 2005. Population growth of monk parakeets in Florida. Florida Field Naturalist, 33, 1-14.

Rabenold, P. P. 1987. Recruitment to food in black vultures: evidence for following from communal roosts. Animal Behaviour, 35, 1775-1785.

Robins, G., Pattison, P. \& Wasserman, S. 1999. Logit models and logistic regressions for social networks: III. Valued relations. Psychometrika, 64, 371-394.

Robins, G., Pattison, P., Kalish, Y. \& Lusher, D. 2007. An introduction to exponentia random graph $\left(p^{*}\right)$ models for social networks. Social Networks, 29, 173-191.

Russello, M., Avery, M. \& Wright, T. 2008. Genetic evidence links invasive monk parakeet populations in the United States to the international pet trade. BMC Evolutionary Biology, 8, 217.

Ryder, T. B., McDonald, D. B., Blake, J. G., Parker, P. G. \& Loiselle, B. A. 2008. Social networks in the lek-mating wire-tailed manakin (Pipra filicauda). Proceedings of the Royal Society B: Biological Sciences, 275, 1367-1374.

Ryder, T. B., Parker, P. G., Blake, J. G. \& Loiselle, B. A. 2009. It takes two to tango: reproductive skew and social correlates of male mating success in a lekbreeding bird. Proceedings of the Royal Society B, 276, 2377-2384.

Sapolsky, R. M. 2005. The influence of social hierarchy on primate health. Science, 308, 648-652.

Silk, J. B. 2007. The adaptive value of sociality in mammalian groups. Philosophical Transactions of the Royal Society B, 362, 539-559.

Silk, J., Alberts, S. \& Altmann, J. 2006a. Social relationships among adult female baboons (Papio cynocephalus) II. Variation in the quality and stability of social bonds. Behavioral Ecology and Sociobiology, 61, 197-204.

Silk, J., Altmann, J. \& Alberts, S. 2006b. Social relationships among adult female baboons (Papio cynocephalus) I. Variation in the strength of social bonds. Behavioral Ecology and Sociobiology, 61, 183-195.
Silk, J. B., Beehner, J. C., Bergman, T. J., Crockford, C., Engh, A. L., Moscovice, L. R. Wittig, R. M., Seyfarth, R. M. \& Cheney, D. L. 2009. The benefits of socia capital: close social bonds among female baboons enhance offspring survival. Proceedings of the Royal Society B, 276, 3099-3104.

de Silva, S., Ranjeewa, A. \& Kryazhimskiy, S. 2011. The dynamics of social networks among female Asian elephants. BMC Ecology, 11, 17.

Snijders, T. A. B., van de Bunt, G. G. \& Steglich, C. E. G. 2010. Introduction to stochastic actor-based models for network dynamics. Social Networks, 32 44-60.

South, J. M. \& Pruett-Jones, S. 2000. Patterns of flock size, diet, and vigilance of naturalized monk parakeets in Hyde Park, Chicago. Condor, 102, 848-854.

Stanton, M. A., Gibson, Q. A. \& Mann, J. 2011. When mum's away: a study of mother and calf ego networks during separations in wild bottlenose dolphins (Tursiops sp.). Animal Behaviour, 82, 405-412.

Sundaresan, S. R., Fischhoff, I. R., Dushoff, J. \& Rubenstein, D. I. 2007. Network metrics reveal differences in social organization between two fission-fusion species, Grevy's zebra and onager. Oecologia, 151, 140-149.

Sundaresan, S. R., Fischhoff, I. R. \& Dushoff, J. 2009. Avoiding spurious findings of nonrandom social structure in association data. Animal Behaviour, 77, 1381-1385.

Tillman, E. A., Genchi, A. C., Lindsay, J. R., Newman, J. R. \& Avery, M. L. 2004 Evaluation of trapping to reduce monk parakeet populations at electric utility facilities. Vertebrate Pest Conference, 21, 126-129.

de Vries, H., Stevens, J. M. G. \& Vervaecke, H. 2006. Measuring and testing the steepness of dominance hierarchies. Animal Behaviour, 71, 585-592.

de Waal, F. B. M. 1986. The integration of dominance and social bonding in primates. Quarterly Review of Biology, 61, 459-479.

Wasserman, S. \& Faust, K. 1994. Social Network Analysis: Methods and Applications. Cambridge: Cambridge University Press.

Wasserman, S. \& Pattison, P. 1996. Logit models and logistic regressions for social networks: I. An introduction to Markov graphs and $p^{*}$. Psychometrika, 61, $401-425$

Wey, T. \& Blumstein, D. 2012. Social attributes and associated performance measures in marmots: bigger male bullies and weakly affiliating females have higher annual reproductive success. Behavioral Ecology and Sociobiology, 66 1075-1085.

Wey, T., Blumstein, D. T. Shen, W. \& Jordán, F. 2007. Social network analysis of animal behaviour: a promising tool for the study of sociality. Animal Behaviour, 75, 333-344.

Whitehead, H. 2008. Analyzing Animal Societies: Quantitative Methods for Vertebrate Social Analysis. Chicago: University of Chicago Press.

Whitehead, H. 2009a. SOCPROG: Programs for Analyzing Social Structure. Version 2.4. Halifax, Nova Scotia: Dalhousie University.

Whitehead, H. 2009b. SOCPROG programs: analysing animal social structures. Behavioral Ecology and Sociobiology, 63, 765-778.

Whitehead, H. \& Dufault, S. 1999. Techniques for analyzing vertebrate socia structure using identified individuals: review and recommendations. Advances in the Study of Behavior, 28, 33-74.

Whitehead, H., Bejder, L. \& Ottensmeyer, C. A. 2005. Testing association patterns: issues arising and extensions. Animal Behaviour, 69, e1-e6. 